[Vicino Oriente XVII (2013), pp. 135-152]

\title{
COSTRUIRE MEMORIA E TRADIZIONE: IL TOFET
}

\author{
Alessandro Campus - Università di Roma "Tor Vergata" \\ The interpretation of the Phoenician-Punic tophet is changed in the years, from the affirmation to \\ the negation of human sacrifice. Beyond the human sacrifice, the tophet can be interpreted as place of \\ the negotiation of the different identities, the Phoenician and the West Phoenician.
}

Keywords: Fenici; Punici; tofet; identità; diaspora

- A morte la romana!

- Che le sue viscere brucino sul petto di Molok!

- Egli ci sarà riconoscente e ci darà novella forza!

- A morte! A morte! Molok vuole vittime nemiche!

Con queste parole inizia il romanzo di Emilio Salgari Cartagine in fiamme, pubblicato a puntate nel settimanale Per terra e per mare nel 1906 e poi in volume nel 1908. Poco importa se poi sarà dimostrata l'inconsistenza del dio Molok $^{1}$, nell'immaginario rimane il collegamento tra i sacrifici umani e Cartagine. Ma l'uso di Molok come divinità avrà vita ancora molto lunga: ad esempio, la II parte della poesia di Allen Ginsberg Howl (1956) è dedicata proprio al "Moloch"; questa è, come esempio, la seconda strofa²:

Moloch! Solitude! Filth! Ugliness! Ashcans and unob-

tainable dollars! Children screaming under the

stairways! Boys sobbing in armies! Old men

weeping in the parks!

Ancora una volta, il "Moloch" - «They broke their backs lifting Moloch to Heaven!», scrive il poeta nel primo verso dell'undicesima strofa - come essere distruttore ${ }^{3}$, che nella immagine del poeta rappresenta la parte peggiore della civiltà umana 4 .

In tal senso, nella prospettiva di (una) definizione della civiltà fenicio-punica, senza dubbio il fenomeno che la storiografia moderna, sulla scorta dei testi biblici, ha definito

1 Eissfeldt 1935. Una storia degli studi è ora in Ribichini 2013. Ma v., ad esempio, la voce moloc nel Vocabolario Treccani, dove, nella versione on line, si può leggere: «propr., nome (ebr. Mōlek, gr. Molovc, lat. Moloch) di un'antica divinità cananea a cui venivano offerte vittime umane; talvolta, in similitudini o in usi fig., indica persona, istituzione, potere e sim. caratterizzati da un'insaziabile sete di distruzione: la gran porta inghiotti, quasi moloch inesorabile, $i$ sopraggiunti (Bonsanti)» (http://www.treccani.it/vocabolario/moloc_res-595a708f-0026-11de-9d89-0016357eee51, 1 settembre 2013). Simile è il testo delle Enciclopedie on line (http://www.treccani.it/enciclopedia/moloc/, 1 settembre 2013). Di fatto, entrambe le voci riprendono il lemma moloc nel volume XXIII della Enciclopedia italiana di scienze, lettere ed arti del 1934, voce redatta da G. Ricciotti (p. 587; questo l'inizio: «Nome di un idolo a cui gli Ebrei del tempo dei re sacrificavano vittime umane nella Valle di Hinnom (Geenna) presso Gerusalemme (II [IV] Re, XXIII, 10; Geremia, XXXII, 35)»); la bibliografia, poi, rimanda al lemma Baal. Negli aggiornamenti successivi della Enciclopedia italiana non c'è traccia di Moloc.

2 Cito da Ginsberg 1986.

3 Scrive J. Raskin (2004, 30): «Moloch murdered Naomi with madness, and Moloch would murder him with madness, too, unless he could slay the monster with the sword of poetry». Naomi era la madre di A. Ginsberg.

4 Secondo il poeta, il verso più importante di Howl è «Moloch whose nameis the/mind!» (cit. da Raskin 2004, 141-142). Sul Moloch di Allen Ginsberg v. Raskin 2004, 130-133, 138-139. 
"tofet" riveste un particolare ruolo ${ }^{5}$. Dal punto di vista della modernità - della contemporaneità -, in quella fluidità che pare caratterizzare letture e riletture di fenomeni antichi $^{6}$, poche culture sono state segnate più nettamente di quella fenicio-punica; così, il tofet è stato visto dagli specialisti di volta in volta come luogo dove venivano compiuti sacrifici di bambini, oppure dove dare pietosa sepoltura ai morti prematuri, o ancora come luogo destinato prioritariamente alle deposizioni di bambini morti di morte naturale e solo marginalmente a riti cruenti ${ }^{7}$.

A livello popolare, invece, la condanna della cultura fenicio-punica è sempre stata netta e senza appello. È sufficiente ricordare la fortuna che ebbe Salammbô, di Gustave Flaubert, o il già citato romanzo di Salgari Cartagine in fiamme . $^{8}$.

È da leggere proprio nell'ottica della risposta all'immagine popolare, ma anche della formazione di tale visione, il racconto di Isaac Asimov The Dead Past, comparso nel numero dell'aprile 1956 della rivista Astounding9. Ambientato in una realtà nella quale è

5 Per una definizione del tofet v. Xella 2010

6 Si veda, ad esempio, il fascicolo 46, 1 (2011) della rivista World Archaeology, interamente dedicato agli studi post-coloniali e alle difficoltà di analisi di situazioni antiche e moderne; di particolare interesse il contributo di van Dommelen 2011. Un altro esempio è quello della cd. 'romanizzazione', per il quale mi permetto di rimandare al mio Campus 2012, in part. il capitolo Una questione di identità, pp. 279-398.

7 V. la storia degli studi - in particolare per quanto riguarda la visione del tofet- delineata da Garnand 2002 (ringrazio l'autore per avermi autorizzato a citare il suo lavoro). Un utile stato della questione è in D'Andrea Giardino 2011. B.K. Garnand pone come discrimine il 1987, l'anno del «ridimensionamento» (in italiano nel testo): in questo anno sono stati pubblicati due saggi da parte di studiosi del mondo fenicio-punico (Ribichini 1987 e Moscati 1987), che appunto 'ridimensionano' il ruolo dei sacrifici cruenti nella religione feniciopunica, ed uno da parte di uno studioso che si considera eccentrico rispetto agli studi classici, M. Bernal (Bernal 1987), che mette in luce la parte che i pregiudizi moderni hanno avuto nelle ricostruzioni del mondo antico.

Trovo significativo, in questa direzione di generale rivalutazione della civiltà fenicio-punica anche tramite la rimozione dei sacrifici di bambini, il volume di E. Mazar, The Northern Cemetery of Achziv (10th-6th centuries BCE) : The Tophet Site, Barcelona 2009, nel quale si usa sin dal titolo la parola tofet; scrive l'autrice nella introduzione: «Since it is customary in Phoenician/Punic studies to identify sites that contained numerous cremation burials as Tophet sites - in this way utilizing the biblical term for the burial ground in Jerusalem where cremations were practiced (...) - this term has also been applied to the case of the Northern Cemetery of Achziv» (p. 21). Il fatto che siano stati ritrovati solo i resti di adulti incinerati non sembra creare un problema per la definizione come "tofet" di questa necropoli. Poi, vengono fatti confronti con la necropoli a incinerazione di al-Bass a Tiro (p. 228) e con i tofet del Mediterraneo occidentale (pp. 229-230). Comunque sia, la fase successiva della necropoli, a partire dal VII sec. a.C. mostra la presenza quasi esclusiva di inumazioni di bambini, con una scarsa presenza di adulti.

Si veda inoltre il caso della necropoli sarda di Su Fraigu, presso Serramanna (CA), in Sardegna, dove un settore dedicato a deposizioni infantili è stato definito dalle studiose 'tofet' (Cossu - Garau 2003, 27-28). Tra l'altro, si tratta di inumazioni e non di incinerazioni.

8 Rimando ancora a Garnand 2002 e a Ribichini 2013.

9 Scrive l'autore, nell'introduzione della raccolta The Best of Asimov (New York 1973): «I had my protagonist interested in Carthage because I myself am a great admirer of Hannibal and have never quite gotten over the Battle of Zama. I introduced Carthage, idly, without any intention of weaving it into the plot. But it got woven in just the same». Una posizione simile, seppur più articolata, è in S. Freud (2011, pp. 196-197): «Nel mio ultimo viaggio in Italia, che tra l'altro mi portò anche sul lago Trasimeno, potei finalmente scoprire, dopo aver preso dolorosamente la via del ritorno, ottanta chilometri prima di Roma, il modo in cui la mia nostalgia per la Città eterna era rafforzata da impressioni infantili. Stavo appunto progettando di fare l'anno dopo un viaggio a Napoli passando per Roma, quando mi colpì una frase, letta forse in uno dei nostri classici: (...) 'Vien da 
necessario chiedere una particolare autorizzazione governativa per poter svolgere le proprie ricerche, questo racconto narra di Arnold Potterley, un professore di storia che chiede di realizzare un chronoscopy, una macchina per vedere il passato ${ }^{10}$, allo scopo di conoscere la verità sui sacrifici umani cartaginesi ${ }^{11}$. Significativamente, è spinto dal rimorso che egli ha per la perdita della figlia, morta nell'incendio della casa dove abitavano.

Nella discussione sul significato del tofet sono recentemente intervenuti alcuni contributi di Corinne Bonnet ${ }^{12}$ e di Josephine Crawley Quinn ${ }^{13}$, le quali cercano di superare il dilemma sacrifici umani/sepolture infantili, per arrivare ad una nuova definizione del tofet, interpretando tale luogo come mezzo per la affermazione dell'identità fenicia d'Occidente.

Per quanto attiene a C. Bonnet, la studiosa mostra come la definizione dell'identità fenicia d'Occidente, nella specifica declinazione storica di "cultura della diaspora", passa anche attraverso la realizzazione di un impianto sacro specifico delle città occidentali ${ }^{14}$. J.C. Quinn, invece, vede nel tofet un luogo nel quale la definizione dell'identità passa attraverso il particolare panorama urbano che il tofet contribuisce a formare. Credo che, seguendo la strada tracciata dalle due studiose, sia possibile percorrere ancora qualche passo verso una definizione di questo spazio.

chiedersi chi abbia passeggiato più nervosamente in camera sua, dopo aver concepito il piano di recarsi a Roma, se il rettore aggiunto Winckelmann o il condottiero Annibale'. Avevo allora seguito le tracce di Annibale; come lui, non ero riuscito a vedere Roma; e anche Annibale era andato in Campania, quando il mondo intero lo aspettava a Roma. Annibale, al quale ero pervenuto a somigliare per questi aspetti, era stato però l'eroe favorito dei miei anni di ginnasio; al pari di molti coetanei, durante le guerre puniche avevo rivolto le mie simpatie non ai Romani ma al Cartaginese. Quando poi, nel ginnasio superiore, capii meglio che cosa vuol dire appartenere a una razza straniera, e l'atteggiamento antisemitico dei miei compagni mi costrinse a prendere una posizione definita, la figura del condottiero semita s'innalzò ancor più ai miei occhi. Annibale e Roma simboleggiavano, per me adolescente, il contrasto fra la tenacia dell'ebraismo e l'organizzazione della Chiesa cattolica, mentre la crescente importanza assunta dal movimento antisemitico sulla nostra vita affettiva contribuiva a fissare i pensieri e i sentimenti di quei lontani giorni. Così il desiderio di andare a Roma è diventato, per la vita del sogno, pretesto e simbolo di molti altri ardenti desideri, la cui realizzazione potrebbe essere perseguita con la costanza e la dedizione del cartaginese, benché il loro appagamento sembri per il momento tanto poco favorito dal destino, quanto la suprema aspirazione di Annibale di entrare in Roma».

10 Dice il protagonista, per giustificare la propria richiesta: «To know Carthage would be very rewarding, yet the only knowledge we have of it is derived from the writings of its bitter enemies, the Greeks and Romans Carthage itself never wrote in its own defense or, if it did, the books did not survive. As a result, the Carthaginians have been one of the favorite sets of villains of history and perhaps unjustly so. Time viewing may set the record straight».

11 Secondo il prof. Potterley, «the Carthaginians, it seemed, worshiped Moloch, in the form of a hollow, brazen idol with a furnace in its belly. At times of national crisis, the priests and the people gathered, and infants, after the proper ceremonies and invocations, were dexterously hurled, alive, into the flames. They were given sweetmeats just before the crucial moment, in order that the efficacy of the sacrifice not be ruined by displeasing cries of panic. The drums rolled just after the moment, to drown out the few seconds of infant shrieking. The parents were present, presumably gratified, for the sacrifice was pleasing to the gods...».

12 Bonnet 2011a; Bonnet 2011b.

13 Quinn 2011.

14 Per le differenti presenze dei tofet nelle varie aree toccate dalla colonizzazione fenicio-punica, rimando a D'Andrea - Giardino 2011, con bibl. prec., in particolare sui concetti di "Circolo dello Stretto" e "Circolo di Cartagine" (così come portati avanti da M.E. Aubet, S. Moscati e P. Bernardini) da una parte e di "Circolo del Tofet", ipotizzato dagli autori, dall'altra. 
Occorre innanzi tutto osservare che, proprio nella definizione dell'identità, il tofet compie - fa compiere - tre passaggi:

negoziazione $\leftrightarrow$ affermazione $\leftrightarrow$ conferma

A livello grafico sarebbe forse meglio rappresentare questi rapporti come un triangolo, nel quale i tre vertici sono i tre passaggi negoziazione, affermazione e conferma, ma con al centro l'identità di Fenici tout court.

\section{NEGOZIAZIONE}

Dal momento che l'identità si forma per contrasto, per differenza, per confronto, è la distanza tra gli abitanti di Madrepatria e i coloni occidentali che marca la diversità tra una definizione orientale ed una occidentale. Identità culturale che non può esser la stessa in Occidente ed in Oriente la cui differenza è possibile per noi cogliere nella ortografia e nella forma delle lettere. M.G. Amadasi $\mathrm{Guzzo}^{15}$ ha individuato le prime attestazioni delle caratteristiche ortografiche puniche, riflesso della pronuncia occidentale, in Sicilia a partire dalla metà del VI sec. a.C.; alla metà - fine del VI sec. a.C. la studiosa fa risalire gli inizi delle forme delle lettere caratteristiche delle iscrizioni occidentali, con una possibile origine a Cartagine di tali forme. È probabile che, al di là del luogo di origine di queste varianti ortografiche e grafiche, si siano diffuse in Occidente da Cartagine, da dove spesso arrivavano magistrati nelle altre città ${ }^{16}$.

Inoltre, la ricerca di una uniformità di paesaggio tra Oriente ed Occidente - ricerca che mi pare più moderna che antica, in verità - porta a definizioni di facies topografiche che allo stato attuale sembrano più linee di tendenza che situazioni costanti nelle scelte insediative

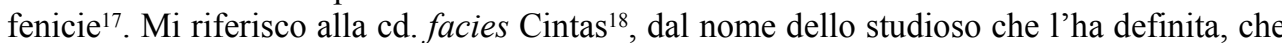
prevede le installazioni fenicie su isole, $\mathrm{o}$ in baie con spiagge riparate, in prossimità di punti d'acqua, di laghi costieri o estuari di fiumi, con alle spalle colline che facciano barriera con l'entroterra. Ma nel Vorwort/Prefazione agli atti del convegno Phönizisches und puniche Städtewesen, S. Helas e D. Marzoli scrivono che, dai dati presentati, si riconoscono solo in parte le vere e proprie concezioni urbanistiche ${ }^{19}$.

\section{AFFERMAZIONE}

Come ha giustamente messo in rilievo Avtar Brah, le domande da porsi per l'analisi del fenomeno della diaspora sono molte ${ }^{20}$ :

"The question is not simply about who travels but when, how, and under what circumstances? What socio-economic, political, and cultural conditions mark the trajectories of these journeys? What regimes of power inscribe the formation of a specific diaspora?».

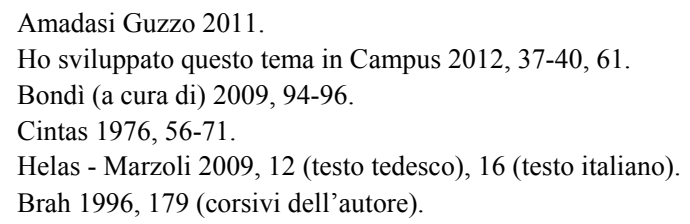


Per lo studioso i temi dell'“arrivo" sono importanti tanto quanto quelli della "partenza": come si conclude il viaggio, ci si insedia in un territorio, si entra in relazione con le culture che si incontrano, in sostanza quanto e come i valori della cultura di provenienza si inseriscono nella prospettiva della nuova sede. Le domande da porsi, quindi, sono anche ${ }^{21}$ :

«How and in what ways do these journeys conclude, and intersect in specific places, specific spaces, and specific historical conjunctures?».

Ciò che importa per A. Brah è la costruzione di un "noi" comune, che si estrinseca storicamente (anche) nella dialettica con le popolazioni che si incontrano. Ma il movimento da un luogo all'altro mette in evidenza anche la dialettica tra due diverse "case", concetto perfettamente delineato nelle parole che il console Lucio Marcio Censorino rivolge agli ambasciatori cartaginesi prima della terza guerra punica ${ }^{22}$ :

eksthtethi Karchdomo hmin, kai; anoikisasqe okh/ qevetethi umetera ogdohkont astadiou ap o; qal assh thudegarhmin egnwstaikat as kay ai.

«Fateci il piacere di abbandonare Cartagine e di emigrare verso l'interno del vostro territorio, nel luogo a vostra scelta, purché sia a 80 stadi dal mare: infatti abbiamo deciso di distruggere la città.».

D'altronde, Livio ricorda che per gli ambasciatori numidi a Roma, in contenzioso nel 162 a.C. con Cartagine per una questione di confini, i Cartaginesi erano stranieri che si erano insediati vi atque iniuria nella zona di Bursa ${ }^{23}$. Così, proprio nell'ottica di una diaspora - quasi che i Fenici fossero "destinati" ad una esistenza nella diaspora, che la loro esistenza si esaurisse con la diaspora - il console del 149 a.C. ricorda che nel passato lasciarono la Fenicia alla volta dell'Africa per costruire una nuova patria; allo stesso modo avrebbero potuto considerare patria il luogo in cui sarebbero andati ${ }^{24}$. Come segno di continuità culturale con Cartagine, i futuri esuli sarebbero potuti tornare nella città, da radere interamente al suolo con l'eccezione dei templi e, significativamente, le tombe, per compiere $\mathrm{i}$ riti $^{25}$. I Romani riconoscono come fondante per la cultura cartaginese l'aspetto rituale, ribaltando propri valori sul nemico, anzi proponendo che la cultura del nemico sia mantenuta tramite l'aspetto rituale, non ritenendo fondamentale l'orizzonte geografico, $\mathrm{o}$, viceversa, ritenendolo talmente fondamentale da volerlo scardinare nel profondo proponendo una nuova sede lontana dal mare. Si propone quindi ai Cartaginesi una seconda diaspora, questa volta però con lo sradicamento globale e la fondazione di un'altra città. Ciò che colpisce è la risposta che gli ambasciatori danno a Censorino, quando questi finisce di esporre le condizioni romane: avendo la certezza di essere uccisi dai Cartaginesi, una volta riferito l'esito dell'incontro, chiedono che la flotta romana li anticipi e si rechi a Cartagine, o meglio, come scrive lo storico, ej i; thnpatrida, quasi una risposta alle "proposte" romane ${ }^{26}$. Si tratta di scegliere una nuova patria, una nuova Madrepatria, che possa rendere accettabile la nuova Cartagine, la nuova "città nuova", alla politica romana.

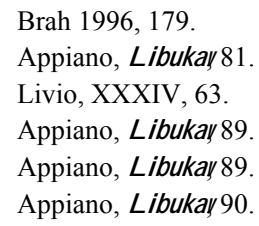


Nell'analisi del movimento coloniale fenicio da Oriente ad Occidente va messa in evidenza la differenza tra la esperienza diasporica fenicia e quelle invece moderne, come ad esempio quella che ha portato milioni di schiavi dall'Africa alle Americhe ${ }^{27}$. Si tratta di una diversità fondamentale per poter comprendere il fenomeno: lo spostamento dei Fenici si configura come un movimento - solo commerciale prima, anche insediativo poi - che non è stato obbligato, ma volontario. Di contro, il trasferimento coatto degli Africani da un luogo all'altro non può non aver costretto ad una profonda riconfigurazione della propria identità, sia personale sia relazionale, nella formazione di una identità collettiva che si forma durante e dopo il viaggio attraverso l'Atlantico, il Middle Passage ${ }^{28}$, dato che gli schiavi portati in Nord America provenivano da aree e da culture africane diverse ${ }^{29}$ : in sostanza, la formazione della nuova identità, che li avrebbe portati da African a African American, doveva passare anche attraverso una cultura altra, ad esempio con l'adozione dell'inglese quale lingua di comunicazione ${ }^{30}$.

Nelle tante slave narratives pubblicate negli Stati Uniti le testimonianze della costruzione delle identità sono esplicite nel mostrare le difficoltà di questo percorso ${ }^{31}$. Una preziosa testimonianza in tal senso è quella relativa ad un rito funebre nella narrative di $\mathrm{C}$. Ball, un ex schiavo; egli descrive il rito per la sepoltura di un bambino, figlio di una schiava nata in Nord America e di uno schiavo catturato in Africa ${ }^{32}$ :

«Her husband, a native of a country far in the interior of Africa, said he had been a priest in his own nation, and had never been taught to do any kind of labour, being supported by the contributions of the public. (...) A few days before Christmas, her child died, after an illness of only three days. I assisted her and her husband to inter the infant which was a little boy - and its father buried with it, a small bow, and several arrows; a little bag of parched meal; a miniature canoe, about a foot long, and a little paddle, (with which he said it would cross the ocean to his own country) a small stick, with an iron nail,

27 Per l'uso del termine “diaspora", v. Peretz 2004, ma anche con i dubbi sull'uso di "diaspora" per il forzato spostamento di Africani attraverso l'Atlantico (v. ad es. a p. 143: «l'utilisation du terme de diaspora, toute comme ultérieurement celle du qualificatif African-American, avait pour ambition de restituer son passé et ses attaches à une communauté qui en avait été dépossédée. Parler de diaspora devait permettre aux Noirs américains de retrouver une part de leur dignité»). V. anche Sideri 2008, per una interessante messa a fuoco della dialettica diaspora/migrazione. Un approccio archeologico alla presenza africana in Nord America è in Campus 2005.

28 Rediker - Pybus - Christopher 2007, 1: «The 'middle passage' is an old maritime phrase, dating to the heyday of the Atlantic slave trade. It designated the bottom line of a trading triangle, between the 'outward passage' from Europe to Africa and the 'homeward passage' from the Americas back to Europe».

29 V. Eltis - Richardson - Behrendt 1999; Yelvington 2005, in part. pp.27-28, tab. 2; Aderinto 2007.

30 Ma v. anche il ruolo della società schiavista nordamericana nel plasmare il concetto di "razza" - e quindi di identità - degli schiavi neri: Berlin 1998, 1-14, 358-365.

31 Per un'analisi di una forte costruzione identitaria, v. i casi di Frederick Douglass, analizzato da Stauffer 2007, e di Venture Smith, analizzato da Pierce 2007, 86-96; quest'ultimo è particolarmente interessante, perché si tratta di una persona catturata in Africa e deportata in Nord America; da notare che il suo nome africano era Broteer (Smith 1798, 5), ma, appunto nell'ottica di una nuova identità (imposta), gli fu cambiato: «I was bought on board by one Robertson Mumford, steward of said vessel, for four gallons of rum, and a piece of calico, and called VENTURE, on account of his having purchased me with his own private venture. Thus I came by my name» (Smith 1798, 13).

32 Ball 1837, 263-265 
sharpened, and fastened into one end of it; and a piece of white muslin, with several curious and strange figures painted on it in blue and red, by which, he said, his relations and countrymen would know the infant to be his son, and would receive it accordingly, on its arrival amongst them. (...) He cut a lock of hair from his head, threw it upon the dead infant, and closed the grave with his own hands. He then told us the God of his country was looking at him, and was pleased with what he had done. Thus ended the funeral service.».

Continuando quindi lungo la strada tracciata da C. Bonnet, "comparando l'incomparabile", come scrive la studiosa ${ }^{33}$ prendendo in prestito il titolo di un volume di M. Detienne ${ }^{34}$, le risposte alle due diaspore qui in esame date dalle rispettive culture si delineano ovviamente come diverse; ma prendendo la descrizione del corredo funerario riportata poco sopra si può notare che la definizione dell'identità è data proprio dagli oggetti che i genitori pongono nella sepoltura del bambino. Il modellino di canoa sarebbe servito al piccolo defunto a tornare alla terra di origine, il pezzo di stoffa sarebbe stato il modo per farsi riconoscere dai parenti e dai connazionali; tutto questo in una prospettiva escatologica. Proprio perché il ritorno all'Africa è in una prospettiva escatologica, quando si tratterà di tornare veramente nel continente il tentativo fallirà, con lo stato della Liberia (indipendente dal 1847) che non diventerà la nuova casa di tutti gli ex schiavi nordamericani e dei loro discendenti ${ }^{35}$.

Rimanendo ancora nell'orizzonte delineato da C. Bonnet - comparando l'incomparabile - il complesso rito del tofet ${ }^{36}$ aveva anche la funzione di riportare le origini occidentali all'Oriente. Si tratta di un Oriente evidentemente non in quanto tale, non luogo geografico o luogo culturale, ma luogo della memoria, memoria che diventa tradizione, proprio nella negoziazione tra le diverse identità d'Oriente e d'Occidente, o, meglio, nella formazione di una nuova identità fenicia, nella possibilità di un ritorno all'Oriente, proprio come l'Africa dove sarebbe dovuto andare il bambino citato da $\mathrm{C}$. Ball era sì il luogo di provenienza del padre, ma era anche un luogo originario. In tal senso, appare ancor più appropriato il confronto - la comparazione - portata avanti dalla studiosa tra il fenomeno tofet ed il candomblé brasiliano ${ }^{37}$.

Angelo Brelich ha mostrato che nell'ambito degli studi storico-religiosi, e in particolare secondo il metodo della "scuola di Roma", la comparazione non serve a mostrare le somiglianze, ma le differenze ${ }^{38}$. Si tratta cioè di mettere in evidenza la differenza delle risposte date alle stesse domande da culture diverse. Pertanto, nella cultura africana della diaspora pare che, almeno nel racconto di C. Ball, il rapporto con la terra di origine sia dato

Bonnet 2011a, 469, 473

4 Detienne 2010.

35 Sulla storia dello stato della Liberia, v. le pagine web nel sito della Library of Congress di Washington (http://www.loc.gov/exhibits/african/afam003.html; http://www.loc.gov/collection/maps-of-liberia-1830-to1870/about-this-collection; http://memory.loc.gov/ammem/gmdhtml/libhtml/libhome.html;).

36 Ribichini 1999-2000; Ribichini 2000; Xella 2012.

37 Bonnet 2011b, 473: «Le candomblé servira de laboratoire pour observer ces éléments à l'oeuvre dans un espace cultuel singulier situé entre racines et migration».

38 Brelich 1972; Brelich 1977. 
dal bambino defunto. Inoltre, il nonno dello scrittore è nato in Africa, come egli stesso scrive ${ }^{39}$ :

«My grandfather was brought from Africa, and sold as a slave in Calvert county, in Maryland, about the year 1730.».

E interessante notare che il racconto di Olaudah Equiano, considerato il fondatore del genere delle slave narratives, inizia dall'infanzia dell'autore in Africa ${ }^{40}$; ebbene, recenti ricerche hanno dimostrato che in effetti egli non nacque in Africa, ma in South Carolina intorno al 1747 , come sono inesatti molti altri particolari che egli racconta ${ }^{41}$. Indubbiamente vi erano motivi politici per attribuirsi origini africane, dato che nel XVIII secolo era prioritaria la campagna di abolizione della tratta degli schiavi (vietata dalla Gran Bretagna e dagli Stati Uniti dal 1808), più che l'abolizione della schiavitù tout court ${ }^{42}$, ma anche la ricerca di una propria identità più "vera" di quella che gli viene attribuita ${ }^{43}$ non può che portarlo verso l'Africa.

\section{CONFERMA}

Se è vero che l'età non è una categoria naturale, ma un concetto culturalmente determinato $^{44}$ e quindi con tante variabili quante sono le possibili culture, con ovvie variazioni diacroniche, allora la scelta di dedicare ai morti in tenera età - ripeto, indipendentemente dal modo in cui sono morti - uno spazio ed un rito separati diventa un fatto culturalmente significativo. Ancora una volta, la preparazione di uno spazio funerario non è in funzione dei morti, ma dei vivi, che costruiscono la propria cultura anche tramite l'attenzione riservata ai defunti. Sono così di grandissimo interesse le osservazioni di K.W. Baptist, la quale a partire dalla propria personale esperienza di lutto scrive $\mathrm{e}^{45}$ :

«Death by landscape. Regardless of the means by which we die, all human remains are returned, in some form, to landscape. (...) Consolation for the living is sought in landscape. Landscape has long provided humans with a physical, sensorial, and ephemeral repository for both grief and for the dead.».

L'elaborazione del lutto si configura in questo modo come la possibilità di ancorare il proprio dolore anche ad un landscape. Di qui il creare o - ri-creare - un orizzonte sia culturale sia fisico proprio nella prospettiva di dare fisicità all'evento.

C'è un ulteriore aspetto, poi, da mettere in evidenza. Com'è noto, intorno alla fine del VI sec. a.C. i riti funerari nella cultura fenicio-punica cambiano, passando dall'incinerazione all'inumazione. Di contro, nel tofet - qualsiasi sia la funzione - i corpi

39 Ball 1837, 15-16

40 Equiano 1789. L'opera di Olaudah Equiano ebbe un grandissimo successo, tanto che durante la vita dell'autore, morto nel 1797, furono stampate ben nove edizioni.

La bibliografia su O. Equiano è vastissima, una sintesi della sua vita, con una bibliografia di riferimento è in Hunt 2007. Una analisi delle sue strategie narrative è in Eckstein 2006, 25-35.

41 Carretta 2007, con bibl. prec.

42 Carretta 2007, 47.

43 Il nome "occidentale" che gli viene attribuito, Gustavus Vassa, è quello dell'eroe svedese nella lotta per l'indipendenza dalla Danimarca.

44 Lucy 2005, in part. pp. 52-61, anche se è da prendere con riserva la parte dedicata al tofet cartaginese.

45 Baptist 2010, 294-295 (corsivi dell'autrice). 
continuano ad essere incinerati. La ricerca della memoria porta alla conferma della tradizione, in uno spazio fisico cui corrisponde uno spazio culturale, con la peculiarità dell'esperienza occidentale della diaspora, che si configura come affermazione contemporaneamente sia della identità fenicia, sia della specificità occidentale. Il trattamento dei corpi deposti nel tofet prosegue la stessa strada "orientale" delle origini, in una continuità che conferma l'uso della Madrepatria, ma che innova rispetto alle origini riservando l'uso solo ad una categoria di persone in uno spazio sacro loro dedicato.

Proprio nel tofet si ha la conferma della propria appartenenza culturale, nel senso di una partecipazione attiva (se si è attore del rito) o passiva (se al rito si assiste, ovvero se si accede all'area). È questo il significato dato alle installazioni di questo tipo da J.C. Quinn, la quale nota come è lo stesso paesaggio urbano della città, segnato dalla presenza del tofet, ad esser significativamente identitario; e questo ${ }^{46}$

«by creating and reinforcing identifications with cultural symbolism and practice elsewhere, within and beyond the Phoenician diaspora.».

Così, il senso di appartenenza o, viceversa, di estraniazione dato all'individuo da questo specifico luogo marca la vicinanza alla - o la distanza dalla - cultura che ha prodotto quell'area. È già la stessa presenza di quest'area a marcare culturalmente la città. Ancora una volta, la differenza tra significante e significato si annulla, per far coincidere questi due versanti: è la presenza, l'esistenza stessa ad esser significativa, dando così ragione delle differenze tra Oriente ed Occidente. Nella continua dialettica tra presente e passato, nel passaggio da memoria a tradizione, o viceversa nella creazione di una specifica tradizione tramite il tofet, da intendersi come spazio fisico ove si compiono riti e come spazio culturale di negoziazione delle diverse identità, è da vedersi il tentativo di assolvere al doppio compito di far sentire contemporaneamente Fenici e Fenici della diaspora. La crisi dell'identità, provocata dal viaggio da Oriente a Occidente e del successivo stanziamento, trova una soluzione nel fenomeno tofet.

Si è soliti mettere il discrimine tra "fenicio" e "punico" al VI sec. a.C., quando, per effetto delle culture di sostrato e di adstrato la cultura fenicia d'Occidente cambia, dando luogo a quella che storiograficamente viene definita "punica" ${ }^{47}$. Ma la cultura fenicia d'Occidente ha verosimilmente iniziato a cambiare subito, nel momento stesso in cui i Tirii hanno iniziato ad insediarsi sulle sponde del Mediterraneo occidentale. E infatti il tofet di Cartagine viene approntato pochi anni dopo la fondazione delle città ${ }^{48}$. Si realizza in tal modo un luogo sacro che diventa specifico delle città occidentali e che nel tempo ne marcherà il paesaggio in modo netto ed inequivocabile. Si cerca cioè di realizzare un dispositivo che possa in qualche modo mettere in azione il meccanismo di ridefinizione della identità, messa in crisi dallo spostamento verso il Mediterraneo occidentale.

Questa chiave di lettura può esser utile nell'interpretare il grande sviluppo che alcuni tofet hanno avuto nel periodo postpunico, come ad esempio quello di Ras Almunfakh

46 Quinn 2011, 390. Per il mondo moderno, sulla creazione di un cultural landscape, v. Francis 2003, articolorecensione a Warner 1959 e Sloane 1951.

47 Moscati 1988. Per una proposta di ridefinizione di sostrati ed adstrati, v. Campus 2012, 12-23.

48 Sulle cronologie della città v. Docter et al. 2004 e Docter et al. 2008. Sul tofet Bénichou-Safar 2004; Ruiz Cabrero 2007, 299-312. 
presso Sabratha, in Libia ${ }^{49}$, o la fondazione di nuovi impianti, come quello di HenchirelHami, in Tunisia ${ }^{50}$. Nel "dramma culturale" del passaggio allo stato romano dopo il 146 a.C., mi pare che lo sviluppo dei tofet risponda alla maggior necessità di affermare la propria cultura, che non è più punica, ma non è neanche romana. Proprio in questo periodo pare esservi maggiormente "bisogno" di costruire e mostrare la propria identità, ricorrendo così a iconografie che indubbiamente hanno elementi di origine sia punica, sia libica, sia romana, ma con un nuovo aspetto che configura queste manifestazioni - percepibili ad esempio nelle stele - come appartenenti ad una nuova cultura, che altrove ho proposto di definire, nel suo divenire storico, "cultura pidgin" e "cultura creola"51. La tensione dialettica tra la cultura romana e la cultura punica (ma anche col fondamentale contributo delle culture libiche) ha come sintesi questa nuova cultura, che si affaccia alla storia come risposta alle nuove istanze che la situazione pone. Nel momento in cui nell'area cambia la prospettiva (culturale, economica, politica, sociale) a seguito della conquista romana, le culture presenti non possono più fornire un orizzonte di riferimento, di qui la necessità di formulare, seppur con tempi indubbiamente lunghi, nuove soluzioni; l'ampliamento del panorama sociale cui sono sottoposte le genti nordafricane obbliga a ripensare interamente il proprio ruolo. L'ipotesi che mi pare di poter avanzare è che il tofet- con le sue manifestazioni sia cultuali sia esteriori (le urne, le stele, i sacelli) - sia da considerarsi, per l'epoca postpunica, la risposta alle trasformazioni che la cultura punica stava subendo. Se è da accettare quanto detto sinora, cioè il ruolo fondamentalmente identitario del tofet, proprio nei momenti di crisi si ha la necessità di riaffermare se stessi tramite questo potente strumento.

G. Woolf ha proposto di interpretare la grande esplosione epigrafica dell'inizio dell'età imperiale romana come reazione alla anxiety che, secondo lo studioso, pervade la società a fronte dei profondi cambiamenti che la investono ${ }^{52}$. Tra l'altro, nota sempre Woolf, le iscrizioni assolvono a tale funzione mostrando ogni aspetto della vita delle persone: parentele, cariche, carriera, nella ricerca di una stabilità che evidentemente la società non dà più. Inoltre, secondo lo studioso «ex-slaves too seem dramatically over-represented in the epigraphy of Rome and other Italian cities» ${ }^{53}$ e questo perché «more generally former slaves had, through their own manumission, experienced social mobility to an extent which must have heightened their sensitivity to the mutable nature of their social identities» ${ }^{54}$. Nelle iscrizioni del tofet, invece, i dati presentati dai testi sono apparentemente piuttosto scarsi: oltre al nome del dedicante, viene solitamente dato anche il patronimico, talvolta seguito dal nome del nonno, talvolta ancora il mestiere ${ }^{55}$. Ma i grandi assenti, gli invisibili, sono proprio i bambini deposti nell'area. Non c'è mai il loro nome, mai si accenna alla loro presenza, quello che conta è il rito che si svolge, all'interno del quale i bambini paiono

49 Taborelli 1992

50 Ferjaoui 2007. Sulle stele, v. Campus 2012, 322-334.

51 Campus 2012, 355-356, 378-382.

52 Woolf 1996; Woolf 2009.

53 Woolf 1996, 35.

54 Woolf 1996, 36

55 Ruiz Cabrero 2008. 
essere più strumenti che protagonisti. È quindi l'intera "vicenda tofet" che è importante; nella ricerca archeologica ciò che rimane è ovviamente solo l'aspetto materiale deposizione ed eventualmente stele - mancando tutta la parte immateriale del rito. Va in ogni caso notato che l'elemento fondamentale del rito del tofet pare esser proprio la deposizione, gli altri elementi sembrano secondari o comunque non fondamentali. Ad esempio, la stele non è sempre presente. Analizzando poi il contenuto delle iscrizioni, appare evidente che si tratta di vere e proprie narratives, che raccontano non la storia dei defunti, ma la storia dei vivi.

Ricordare i morti per onorare i vivi: lo scopo delle iscrizioni, che, come detto, non nominano mai i defunti deposti nell'area, servono ad ancorare socialmente e culturalmente i dedicanti, in una rete di rapporti genealogici e sociali. Leggendo i testi, si delinea un grande mosaico nel quale prendono posto tutti gli elementi necessari ad identificare le singole persone; attraverso le epigrafi del tofet si propone un racconto autobiografico, il cui protagonista assoluto è chi ha compiuto il rito. Le divinità citate, Ba'al Hammon da solo nelle iscrizioni più antiche poi con Tanit, nella sua particolare manifestazione di pnb'l, sono gli altri protagonisti del rito; talvolta si sente la necessità di specificare anche il rito svolto nell'area - $m l k, m l k ' m r, m l k$ ' $d m$, $m l k b^{\prime} l$ - probabilmente sintomo del fatto che non sempre erano scontate le modalità di svolgimento; alla fine dell'iscrizione spesso è indicato il motivo della dedica, con formule come ad esempio $k$ šm 'ql'ybrk' oppure brk', "poiché ha ascoltato la sua voce lo benedica" o "lo ha benedetto", o $k$ šm'qldbry, "poiché ha ascoltato la voce delle sue parole". Queste iscrizioni, quindi, si configurano come forme comunicative, estremamente complesse nonostante la loro apparente semplicità e standardizzazione, rivolte sì alla o alle divinità, ma anche - e soprattutto - alle altre persone della comunità. È significativo che tra le stele si trovino esempi di monumenti solo figurati, solo iscritti, figurati e iscritti, né figurati né iscritti. Un caso particolare è quello delle stele recanti solo l'iscrizione, senza alcun simbolo o decorazione; tale tipo di monumenti, analizzato qualche tempo fa da M.L. Uberti ${ }^{56}$, evidentemente privilegia un tipo di comunicazione particolarmente specialistico, quale è quello della scrittura.

Nelle dinamiche comunicative, quindi, ciò che maggiormente conta è la presenza, al di là poi di quel che reca il supporto. Anzi, l'aspetto maggiormente significativo - l'unico aspetto che ha veramente valore - è l'urna con i resti del bambino o dell'animale. Infatti, quando ad esempio si è proceduto con la risistemazione del tofet di Mozia sono state le stele ad esser asportate e riutilizzate nella costruzione del terrapieno, mentre i vasi sono stati lasciati in situ ${ }^{57}$. Tra l'altro, proprio a Mozia è interessante vedere il rapporto numerico tra il totale delle stele e le stele iscritte: su un totale di $1185^{58}$ stele solo 40 recano una

56 Uberti 2003.

57 Una sintesi delle vicende del tofet moziese, con bibliografia precedente, è in Nigro 2009, 252-254.

58 Moscati - Uberti 1981; a questi 1185 esemplari editi nel catalogo, che comprende sia quelli degli scavi 19641973 che quelli della collezione Whitaker, sono da aggiungere altre tre stele, ritrovate al di fuori dell'area del tofet (Moscati 1986; Spanò Giammellaro 2000; De Simone 2012). Scrive M.L. Uberti (Moscati - Uberti 1981, 75): «rimangono esclusi pochi esemplari, ancora in situ nella trincea 36, ed ora protetti da una bassa tettoia, che ne rende estremamente difficile l'accesso e dei quali si davano alcuni appunti preliminari nel rapporto relativo alla campagna di scavo 1972» (Uberti 1973, 86, nota 5; Ciasca 1978, 126, nota 2). La studiosa (Uberti $1973,85)$ scrive che nella trincea 36 sono state lasciate in situ 65 stele, mentre A. Ciasca $(1978,126$, nota 2) 
iscrizione, pari al 3,4\%. I testi, poi, hanno la dedica al solo Ba'al Ḥammon, non è presente Tanit ${ }^{59}$.

Rimanendo al tofet dell'isola siciliana, i lavori di A. Ciasca hanno mostrato come solo dalla seconda fase dell'impianto si è incominciato ad usare le stele come segnacolo - sema del luogo di deposizione dell'urna. Appare quindi evidente che la centralità del rito è da ricercarsi proprio nelle fasi che hanno preceduto le deposizioni, fasi delle quali a noi rimane solo l'ultima manifestazione materiale. Inoltre, le analisi hanno mostrato come la deposizione di ceneri di piccoli animali, quali volatili, non sia un uso più recente rispetto a quello di deporre le ceneri di bambini, ma anzi si trova sin dagli strati più antichi ${ }^{60}$. Ciò che conta, ciò che ha valore è il fatto di essersi recato nel tofet per svolgere il rito, sia esso relativo ad un bambino o ad un animale.

Per quanto sin qui detto, credo di aver mostrato quindi che il rito del tofet, oltre che il tofet stesso, è un segno di appartenenza alla comunità; in tal senso, il segno esteriore - il tofet con i suoi annessi - come metafora della "fenicità" d'Occidente. Se questo è vero per quanto riguarda il momento successivo alla "invenzione" del tofet, bisogna porsi il problema delle fasi precedenti tale invenzione. Cioè, perché il tofet?

$\grave{E}$ ancora C. Bonnet che propone una interessante risposta a questa domanda; secondo la studiosa, in una società coloniale così ristretta come doveva esser quella delle prime fondazioni fenicie, la morte di un bambino, tutt'altro che infrequente vista la grande mortalità infantile nel mondo antico ${ }^{61}$, poteva metter a rischio la sopravvivenza stessa della comunità $^{62} \cdot \operatorname{Cosi}^{63}$

«Si le tophet a bien été institué dans les tout premiers temps de la colonie punique, on peut formuler l'hypothèse qu'il constituait une réponse «religieuse» à une telle crainte. Baal Hammon et Tanit, les destinataires des offrandes, comme l'ont bien montré diverses études récentes (...), sont de fait des divinités originaires de Phénicie, bienveillantes et protectrices, et non des monstres assoiffés de sang. On les concevrait volontiers comme des parents attentifs à leur descendance.».

Ecco che quindi il luogo di sepoltura infantile diventa luogo dell'identità e della memoria, nella risoluzione delle diverse tensioni che di volta in volta si alternano nella vita delle città fenicio-puniche.

Ancora "comparando l'incomparabile", l'interessante lavoro di Chiara Garattini sui cimiteri infantili dell'Irlanda moderna e contemporanea ${ }^{64}$ mostra come sino a pochi decenni fa si tendesse a seppellire i bambini morti prima del battesimo o i feti in aree riservate, in linea di massima al di fuori degli altri cimiteri: nei campi, o agli incroci della strade, o in

scrive che «sono state rimosse urne e stele che gli slittamenti del terreno non consentivano più di ritenere effettivamente in situ», ma senza specificare quante stele.

59 Amadasi Guzzo 1986, 45; osserva la studiosa che le stele iscritte e le stele in genere compaiono solo sino al livello III, non oltre quindi gli inizi del V sec. a.C. (Amadasi Guzzo 1986, 45, nota 2).

60 Bernardini 2002, in part. p. 20. Sulle analisi ai contenuti delle urne provenienti da vari siti, si veda la bibliografia in Docter et al. 2001-2002.

61 Woods 2007.

62 Bonnet 2011a, 479-480.

63 Bonnet 2011a, 480

64 Garattini 2007. 
aree vicine a cimiteri, e sempre in forma anonima. Questi killeens (o cillíní, in irlandese) sono caratterizzati da una non riconoscibilità nel paesaggio e dalla mancanza di elementi utili ad identificare le tombe. La esistenza di queste aree era associata alla credenza nel Limbo, il luogo in cui sarebbero andati, secondo gli insegnamenti della chiesa cattolica, i bambini morti senza aver ricevuto il battesimo. Quando, agli inizi dell'Ottocento, la chiesa cattolica iniziò una nuova politica di presenza attiva in Irlanda, fondando nuove chiese e cimiteri, di fatto riservati ai soli cattolici, all'interno dei quali furono accolti anche i corpi dei bambini morti non battezzati. In particolare, nel cimitero di Glasnevil una parte dell'area, ora nota come Old Holy Angels, fu dedicata alle sepolture infantili; negli anni Venti del XX secolo fu aperta una nuova area per i bambini, detta New Holy Angels. La situazione delle sepolture rimase simile a quella dei killeens, con sepolture comuni e non distinte, ma a partire dagli anni Settanta si iniziò individuare alcune sepolture e a mettere oggetti vicino alle tombe. Ora, nonostante l'amministrazione abbia cercato di regolarizzare la situazione, i genitori dei bambini lasciano sulle tombe una enorme quantità di fiori, giocattoli, come doni. Scrive la studiosa ${ }^{65}$ :

«In the past in Ireland (and not only there), the event of an infantile death was silenced in people's lives never to be mentioned again even between spouses. This ability to forget, which was a strategy perceived as the best thing to do, is now in modern eyes seen as unhealthy.».

La specificità fenicio-punica sta probabilmente qua: nella capacità e volontà di non dimenticare, di ricordare la morte dei propri bambini, mostrandola in modo netto e inequivocabile, contrariamente a quanto invece si fece a Roma ${ }^{66}$.

Si tratta, come detto, di una morte che investe l'intera società e che l'intera società deve quindi risolvere. Proprio perché la morte viene "utilizzata" in chiave identitaria, ciò che conta è che i genitori portino il bambino all'interno dell'area del tofet e che compiano i giusti riti; nelle iscrizioni poi è ricordato il genitore che ha compiuto il rito, perché l'identità da riaffermare è di chi è sopravvissuto.

\section{BIBLIOGRAFIA}

ADERINTO, S.

2007 Ethnicity: T. FAlOLA - A. WARNOCK (eds.), Encyclopedia of the Middle Passage, Westport - London 2007, pp. 165-166.

AMAdASI GuZzo, M.G.

$1986 \quad$ Scavi a Mozia - le iscrizioni, Roma 1986.

2011 On the Beginnings of the Punic Scripts: Vicino \& Medio Oriente XV (2011), pp. 119-132.

BALL, $\mathrm{CH}$.

1837 Slavery in the United States. A Narrative of the Life and Adventures of Charles Ball, a Black Man, Who Lived Forty Years in Maryland, South Carolina and Georgia, as a Slave under Various Masters, and Was One Year in the Navy with Commodore Barney, during the Late War, New York 1837.

65 Garattini 2007, 203.

66 Campus 2010. 


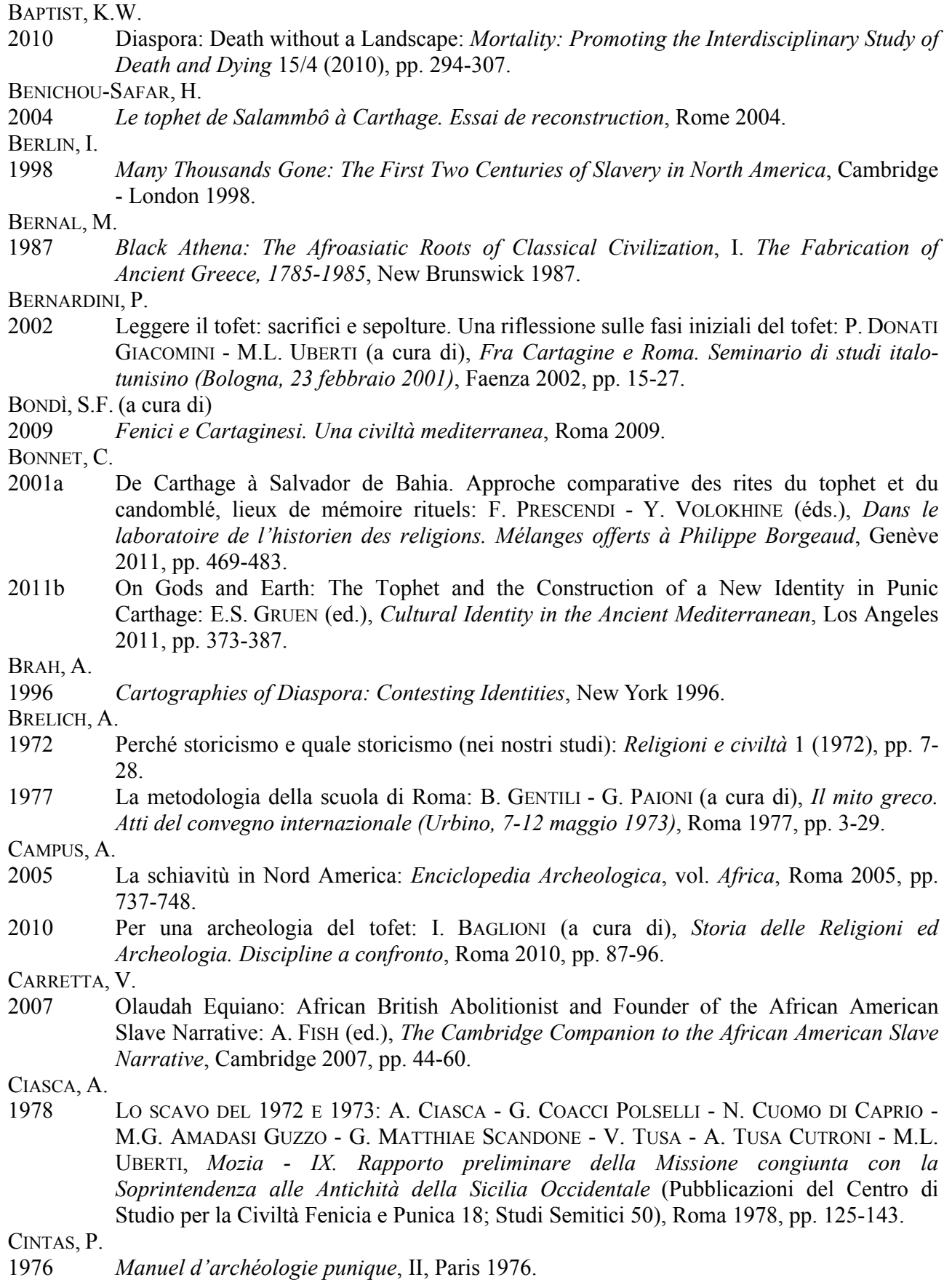

2001a De Carthage à Salvador de Bahia. Approche comparative des rites du tophet et du candomblé, lieux de mémoire rituels: F. Prescendi - Y. Volokhine (éds.), Dans le laboratoire de l'historien des religions. Mélanges offerts à Philippe Borgeaud, Genève 2011, pp. 469-483.

2011b On Gods and Earth: The Tophet and the Construction of a New Identity in Punic Carthage: E.S. GRUEN (ed.), Cultural Identity in the Ancient Mediterranean, Los Angeles 2011, pp. 373-387.

BRAH, A.

1996 Cartographies of Diaspora: Contesting Identities, New York 1996.

BRELICH, A.

1972 Perché storicismo e quale storicismo (nei nostri studi): Religioni e civiltà 1 (1972), pp. 7 28.

1977 La metodologia della scuola di Roma: B. GentiLI - G. PAIONI (a cura di), Il mito greco. Atti del convegno internazionale (Urbino, 7-12 maggio 1973), Roma 1977, pp. 3-29. CAMPUS, A.

2005 La schiavitù in Nord America: Enciclopedia Archeologica, vol. Africa, Roma 2005, pp. 737-748.

2010 Per una archeologia del tofet: I. Baglioni (a cura di), Storia delle Religioni ed CARretta, V. Archeologia. Discipline a confronto, Roma 2010, pp. 87-96.

2007 Olaudah Equiano: African British Abolitionist and Founder of the African American Slave Narrative: A. FisH (ed.), The Cambridge Companion to the African American Slave Narrative, Cambridge 2007, pp. 44-60.

CIASCA, A.

1978 Lo scavo del 1972 e 1973: A. Ciasca - G. Coacci Polselli - N. CuOmo di Caprio M.G. Amadasi Guzzo - G. Matthiae Scandone - V. Tusa - A. Tusa Cutroni - M.L. UberTI, Mozia - IX. Rapporto preliminare della Missione congiunta con la Soprintendenza alle Antichità della Sicilia Occidentale (Pubblicazioni del Centro di CINTAS, P Studio per la Civiltà Fenicia e Punica 18; Studi Semitici 50), Roma 1978, pp. 125-143.

1976 Manuel d'archéologie punique, II, Paris 1976. 
Cossu, C. - GARAu, E.

2003 Complessità rituale e ideologia funeraria punica nella necropoli di Su Fraigu (Serramanna - CA): Soprintendenza archeologica per le province di Cagliari e Oristano. Quaderni 20 (2003), pp. 17-45.

D'ANDREA, B. - GIARDINO, S.

2011 "Il tofet dove e perché": alle origini dell'identità fenicia: Vicino \& Medio Oriente XV (2011), pp. 133-157.

DE Simone, $R$

2012 Una nuova stele da Mozia: C. Del VAIS (a cura di), Epi oinopa ponton. Studi sul DETIENNE, M Mediterraneo antico in memoria di Giovanni Tore, Oristano 2012, pp. 315-319.

$2010 \quad$ Comparer l'incomparable, Paris $2010^{2}$.

DOCTER, R.F. - SMits, S. -HAKBIJL, T. - STUIJTS, I.L.M. - VAN DER Plicht, J.

2001-2002 Interdisciplinary Research on Urns from the Carthaginian Tophet and Their Contents: Palaeohistoria 43-44 (2001-2002), pp. 417-433.

VAN DOMMELEN, $\mathrm{P}$.

2011 Postcolonial Archaeologies between Discourse and Practice: World Archaeology 46/1 (2011), pp. 1-6.

ECKSTEIN, L.

2006 Re-Membering the Black Atlantic. On the Poetics and Politics of Literary Memory, Amsterdam - New York 2006.

EISSFELDT, O

1935 Molk als Opferbegriff im Punischen und Hebräischen und das Ende des Gottes Moloch (Beitrage zur Religionsgeschichte des Altertums 3), Halle 1935.

ELTIS, D. - RichARDSON, D. - BEHRENDT, S.D.

1999 Patterns in the Transatlantic Slave Trade, 1662-1867: New Indications of African Origins of Slaves Arriving in the Americas: M. Diedrich - H.L. GATES - C. Pedersen (eds.), EQUIANO, O Black Imagination and the Middle Passage, New York - Oxford 1999, pp. 21-32.

1789 The Interesting Narrative of the Life of Olaudah Equiano, or Gustavus Vassa, the African. FERJAOUI, A. Written by Himself, London 1789

2007 Le sanctuaire de Henchir el-Hami. De Ba'al Hammon au Saturne Africain, ${ }^{e r}$ s. av. J.-C. - IV ap. J.-C., Tunis 2007.

FRANCES, D.

2003 Cemeteries as Cultural Landscapes: Mortality: Promoting the Interdisciplinary Study of FREUD, S. Death and Dying 8/2 (2003), pp. 222-227.

2011 L'interpretazione dei sogni, edizione integrale (traduzione E. Fachinelli - H. Trettl), Torino 2011.

GARNAND, B.

2002 From Infant Sacrifice to the ABCs: Ancient Phoenicians and Modern Identities: Stanford $\begin{array}{llllll}\text { Journal of } & \text { Archaeology, } & 1 & \text { (2002), } & \text { pp. }\end{array}$ (http://www.stanford.edu/dept/archaeology/journal/newdraft/garnand/paper.html).

GinsBERG, A.

1986 Howl: Original Draft Facsimile, Transcript \& Variant Versions, Fully Annotated by Author, with Contemporary Correspondence, Account of First Public Reading, Legal Skirmishes, Precursor Texts \& Bibliography, New York 1986. 


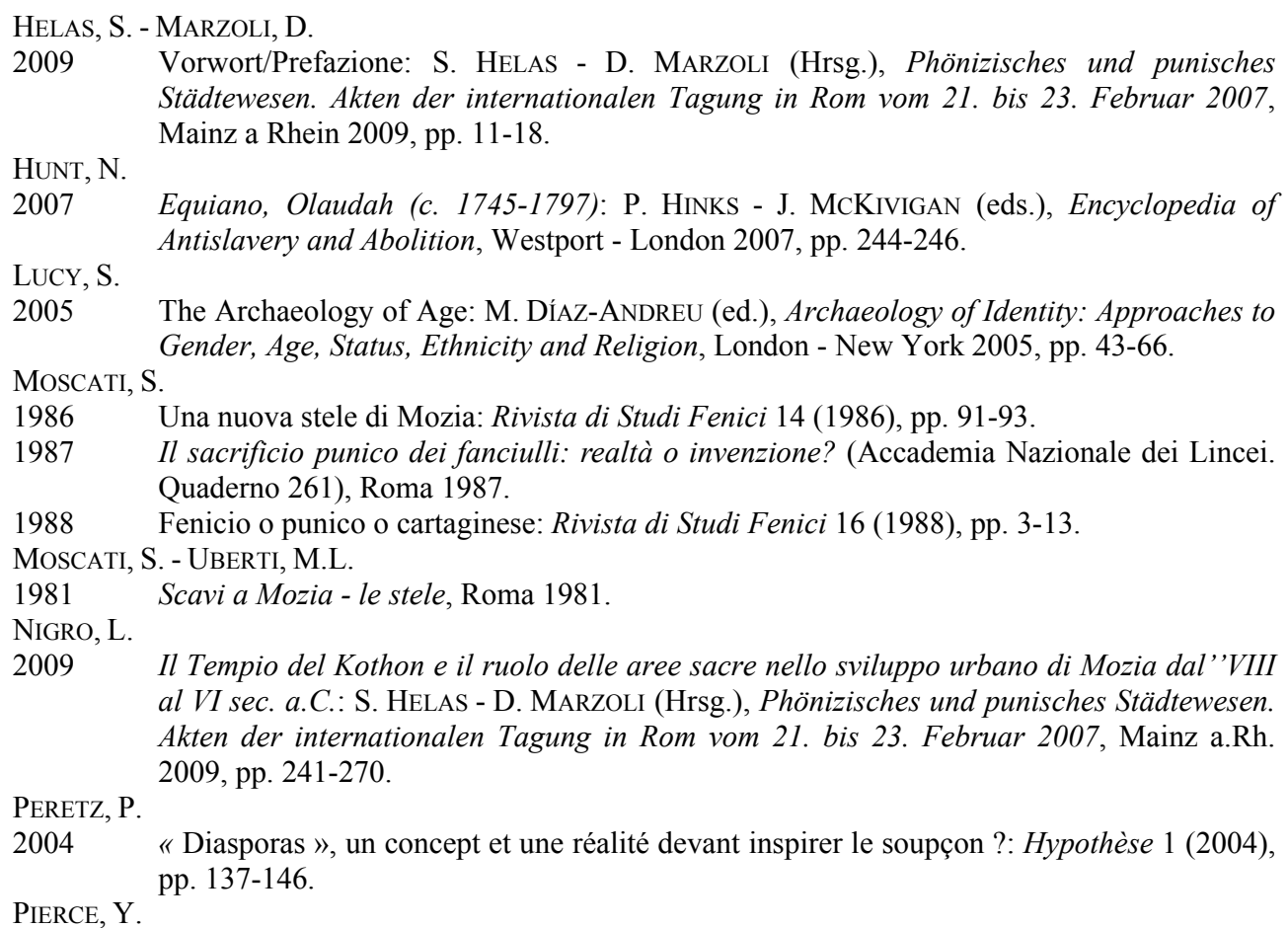

2007 Redeeming Bondage: The Captivity Narrative and the Spiritual Autobiography in the African American Slave Narrative Tradition: A. FisH (ed.), The Cambridge Companion to QUINN, J.C. the African American Slave Narrative, Cambridge 2007, pp. 83-98.

2011 The Cultures of the Tophet: Identification and Identity in the Phoenician Diaspora: E.S. Gruen (ed.), Cultural Identity in the Ancient Mediterranean, Los Angeles 2011, pp. 388413.

RASKIN, J.

2004 American Scream: Allen Ginsberg's Howl and the Making of the Beat Generation, Berkeley - Los Angeles - London 2004.

RediKer, M. - Pybus, C. - Christopher, E.

2007 Introduction: M. ReDIKER - C. Pybus - E. Christopher (eds.), Many Middle Passages: Forced Migration and the Making of the Modern World, Berkeley - Los Angeles RIBICHINI, S. London 2007, pp. 1-19.

$1987 \quad$ Il tofet e il sacrificio dei fanciulli, Sassari 1987.

1999-2000 Sacrum magnum nocturnum. Note comparative sul molchomor nelle stele di N'Gaous: Aula Orientalis 17-18 (1999-2000) = M. Molina - I. MÁRQuez Rowe - J. SANMARTín (edd.), Arbor scientiae. Estudios del Próximo Oriente Antiguo dedicados a Gregorio del Olmo Lete con ocasión de su 65 aniversario, pp. 353-362.

2000 La questione del tofet punico: S. VERGER (ed.), Rites et espace en pays celte et méditerranéen. Étude comparée à partir du village d'Acy-Romance (Ardennes/France). Actes du Colloque international (Rome, 18-19 avril 1997), Rome 2000, pp. 293-304. 
2013 Histoires de Moloch, le roi effroyable: À.A. NAGY - F. Prescendi (éds.), Sacrifices humains. Dossiers, discours, comparaisons. Actes du colloque tenu à l'Université de Genève, 19-20 mai 2011, Turnhout 2013, pp. 209-230.

RUIZ CABRERO, L.A.

2007 El sacrificio molk entre los Fenicios-púnicos: cuestiones demográficas y ecológicas, Madrid 2007.

2008 Dedicantes en los tofet: la sociedad fenicia en el Mediterráneo: Gerión, 26/1 (2008), pp. 89-148.

SIDERI, E.

2008 The Diaspora of the Term Diaspora: A Working-Paper of a Definition: Transtext(e)s SLOANE, D.C.

1991 The Last Great Necessity: Cemeteries in American History, Baltimore 1991.

SMITH, V.

1798 A Narrative of the Life and Adventures of Venture, a Native of Africa: But Resident above Sixty Years in the United States of America. Related by Himself, New London 1798.

SPANÒ GIAMMELlaro, A.

2000 Scavi nella "zona K" di Mozia. L'ottava campagna di scavo (Maggio-Luglio 1994): i materiali: M.E. Aubet, M. BARThÉLEMy (eds.), Actas del IV Congreso Internacional de Estudios fenicios y púnicos (Cádiz, 2 al 6 de Octubre de 1995), Cádiz 2000, III, pp. 1377 1395 .

STAUFFER, J.

2007 Frederick Douglass's Self-Fashioning and the Making of a Representative American Man: A. Fish (ed.), The Cambridge Companion to the African American Slave Narrative, Cambridge 2007, pp. 201-217.

TABORELLI, L.

1992 L'area sacra di Ras Almunfakh presso Sabratha. Le stele, (Rivista di Studi Fenici XX supplementum 2), Roma 1992.

UBERTI, M.L.

1973 Note sulle stele: A. CIASCA - V. TUSA - M.L. UbERTI, Mozia - VIII. Rapporto preliminare della Missione congiunta con la Soprintendenza alle Antichità della Sicilia Occidentale (Pubblicazioni del Centro di Studio per la Civiltà Fenicia e Punica 14; Studi Semitici 45), Roma 1973, pp. 85-91.

2003 Iconismo e scrittura: P. DONATI GiACOMini - M.L. UberTi (a cura di), Fra Cartagine e Roma, II. Secondo Seminario di studi italo-tunisini, Faenza 2003, pp. 5-27.

WARNER, W.L.

1959 The Living and the Dead: A Study of the Symbolic Life of Americans, New Haven 1959.

WoODs, R.

2007 Ancient and Early Modern Mortality: Experience and Understanding: The Economic History Review 60/2 (2007), pp. 373-399.

WOOLF, G.

1996 Monumental Writing and the Expansion of Roman Society in the Early Empire: Journal of Roman Studies 86 (1996), pp. 22-39.

2009 Literacy or Literacies in Rome?: W.A. JOHNSON - H.N. PARKER (eds.), Ancient Literacies: YELVINGTON, K.A.

The Culture of Reading in Greece and Rome, Cambridge 2009, pp. 46-68.

2005 African Diasporas in the Americas: M. EMBer - C.R. EMBer - I. Skoggard (eds.), Encyclopedia of Diasporas. Immigrant and Refugee Cultures in the World, New York 2005, I, pp. 24-35. 
Xella, P.

2010 Per un "modello interpretativo" del tofet: il tofet come necropoli infantile?: G. BARTOLONI - P. Matthiae - L. Nigro - L. Romano (a cura di), Tiro, Cartagine, Lixus: nuove acquisizioni. Atti del Convegno Internazionale in onore di Maria Giulia Amadasi Guzzo (Roma, 24-25 novembre 2008), (Quaderni di Vicino Oriente IV), Roma 2010, pp. 259278.

2012 Il tophet. Un'interpretazione generale: S. ANGiolillo - M. GiUMAN - C. Pilo (a cura di), Meixis. Dinamiche di stratificazione culturale nella periferia greca e romana. Atti del Convegno Internazionale di Studi "Il sacro e il profano" (Cagliari, Cittadella dei Musei 3-7 maggio 2011), Roma 2012, pp. 1-17. 